\title{
DUST IN DENSE CLOUDS
}

\author{
A. G. G. M. TiELENS \\ Space Sciences Division, NASA Ames Research Center, CA 94095 \\ and \\ Space Sciences Laboratory, UC Berkeley, CA 94720
}

\begin{abstract}
Recent observational and theoretical studies of dust in dense clouds are reviewed with an emphasis on the growth of dust grains through accretion and coagulation. IR reflection nebulae around protostellar objects are useful probes of grain sizes in dense clouds. For example, detailed studies of the IR reflection nebula surrounding OMC 2-IRS 1 show that the (scattering) grains are much larger $(\approx 5000 \AA)$ than in the diffuse interstellar medium. Likewise, the presence of a weak shoulder at $2.95 \mu \mathrm{m}$ on the $3.08 \mu \mathrm{m}$ feature in $\mathrm{BN}$ indicates the importance of scattering by icy grains and implies a very similar increase in the grain size.
\end{abstract}

Theoretical studies of grain surface chemistry predict the possible presence of three distinctly different grain mantle components in dense clouds depending on the physical conditions in the gas phase. These are: 1) A hydrogenated mantle dominated by $\mathrm{H}_{2} \mathrm{O}$ and $\left.\mathrm{CH}_{3} \mathrm{OH} ; 2\right)$ An inert grain mantle dominated by $\mathrm{CO}$ and $\mathrm{O}_{2}$; and 3) An oxidized grain mantle dominated by $\mathrm{CO}_{2}$. Although the importance of $\mathrm{H}_{2} \mathrm{O}$ dominated grain mantles was known for $10 \mathrm{yrs}$, the presence of $\mathrm{CH}_{3} \mathrm{OH}$ was only recently confirmed. Furthermore, recent studies of the solid $C O$ band have revealed the presence of at least two distinctly different interstellar grain mantle components along the line of sights towards most stars: One dominated by polar and one by non-polar molecules. Although specific identification of the molecules mixed in with the $C O$ in these components is difficult, it is quite possible that the former component is dominated by $\mathrm{H}_{2} \mathrm{O}$ and the latter by $\mathrm{CO}$ itself, as suggested by theoretical models. Finally, the photochemical evolution of icy grain mantles is briefly reviewed and it is suggested that the resulting complex molecular mantles may evolve into amorphous carbon mantles in the diffuse ISM.

Grain-grain collisions can lead to large modifications of the interstellar grain size distribution. At high velocities $\left(v \gtrsim 1 \mathrm{~km} \mathrm{~s}^{-1}\right)$ shattering into many small fragments will be important, while at low velocities $\left(v \lesssim 10 \mathrm{~m} \mathrm{~s}^{-1}\right)$ coagulation dominates. Both processes can play a role in dense molecular clouds. The sticking of grains at low velocities is discussed in some detail and it is concluded that coagulation in molecular clouds is only important if the colliding grains are covered by icy grain mantles.

Thus, a model for interstellar dust is proposed in which small ( $\lesssim 500 \AA$ ) silicate and carbonaceous grains are "glued" together in large $(\approx 3000 \AA)$, open conglomerates by a polymerized, all enveloping grain mantle. This structure resembles that of certain interplanetary dust particles collected in the upper stratosphere. 


\section{INTRODUCTION}

There are many independent indications that grain growth is an important process inside dense molecular clouds. First, many objects associated with molecular clouds show a steepening of the visual extinction curve as well as an increase in the wavelength of maximum polarization (cf., Whittet and van Breda, 1978; Serkowski, Mathewson and Ford, 1975). These are generally attributed to the presence of larger than average grains. The good correlation between these two independent indicators of grain size further strengthens this interpretation. The observed variations are rather large and imply a size increase by about $50 \%$ in dense clouds for the grains responsible for the visual and NIR extinction and polarization. Second, objects embedded in, or located behind, dense clouds show infrared absorption features at $3.08,4.67,6.0$, and $6.85 \mu \mathrm{m}$ due to molecules in a newly accreted icy grain mantle. These features are absent in objects with only diffuse interstellar dust along the line of sight (cf., Tielens and Allamandola, 1987a). Third, gas phase abundances of heavy elements decrease with increasing gas density, presumably due to accretion on grain surfaces (cf., Jenkins, 1989). Fourth, for two sources embedded in the $\rho$ Oph molecular cloud the amount of visual extinction per $H$ atom is less than in the diffuse interstellar medium (Shull and van Steenberg, 1985; de Boer et al., 1986), suggesting that grain growth due to coagulation has taken place (Jura, 1980). Finally, bright near-IR reflection nebulae are common phenomena around embedded (proto)stars. The implied high NIR albedo also indicates the presence of large grains (Rouan and Léger 1984; Pendleton, 1987).

Several processes can modify the average grain size in dense clouds. First, gas phase species can accrete and react on a grain surface forming a molecular ice mantle consisting of simple molecules such as $\mathrm{H}_{2} \mathrm{O}, \mathrm{CO}, \mathrm{NH}_{3}$, and $\mathrm{CH}_{3} \mathrm{OH}$ (Tielens and Hagen, 1982). These icy grain mantles can be transformed into more complex molecular grain mantles by UV photolysis producing free radicals and transient heating promoting diffusion and reaction of those radicals (Greenberg, 1979). Probably, prolonged exposure to UV photons will eventually drive off most of the other elements than carbon, leading to the formation of an amorphous carbon grain mantle (van de Bult, 1982). Second, the size distribution of interstellar grains will be affected by grain-grain collisions. At low velocities $(v \lesssim 10 \mathrm{~m} / \mathrm{s})$ such collisions will lead to grain growth through coagulation, while at high velocities $(v \gtrsim 1$ $\mathrm{km} \mathrm{s}^{-1}$ ) shattering into many small fragments becomes important. Third, observations show that many protostars have strong stellar winds, which give rise to shocks in the surrounding material. In such shocks, grains can be destroyed by sputtering due to high velocity, ion impacts. They can also be vaporized and shattered by grain-grain collisions (cf., McKee, 1989). Finally, the high densities associated with star formation may lead to dust condensation (and planet formation) in the outflow or accreting protostellar disk. Such newly formed dust could be injected into the surrounding molecular cloud by a protostellar wind.

In summary, many independent observations indicate that grains are larger in dense clouds than in the diffuse interstellar medium and that this results from a number of processes, including the formation of icy grain mantles as well as grain agglomeration. In this paper our knowledge is reviewed with an emphasis on recent developments. $\$ 2$ discusses IR studies of reflection nebulae and their implications for grain sizes in dense clouds. $\S 3$ confronts theoretical studies of grain surface chemistry with (recent) observational studies of interstellar icy grain mantles. $§ 4$ 


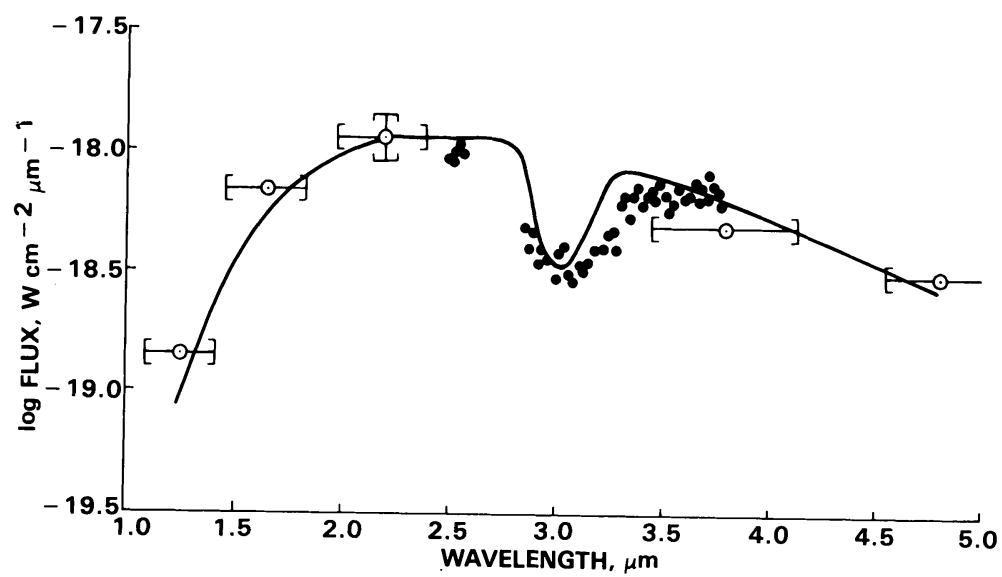

Fig. 1. An isotropic scattering model using the MRN dust model (solid line) is compared to the observations (dots) of the reflection nebula surrounding OMC 2 IRS 1 (Pendleton et al., 1989). This is however not an unique fit and larger grains may explain the observations as well. Indeed, polarization studies indicate the dominance of larger grain $(a \simeq 5000 \AA)$.

discusses the photochemical evolution of ice grain mantles into organic refractory grain mantles and ultimately amorphous carbon. $\$ 5$ considers the collisional agglomeration of small grains. §6 presents some speculation on the influence of star formation on interstellar grains. Finally, the results are summarized in $\S 7$.

\section{IR REFLECTION NEBULAE AROUND PROTOSTARS}

Many protostars have associated bright, extended, near-infrared reflection nebulae. The Kleinman-Low nebula in the Orion Molecular Cloud is a good example, since polarization studies have shown that most of its near-infrared radiation is due to scattering of photons from the embedded protostars OMC1-IRC2 and BN by dense clumps (Werner et al., 1983). Clearly, infrared studies of reflection nebulae can be a very useful tool for probing the structure of star-forming regions. Such studies may also provide information on dust grain properties in star forming regions. Indeed, it has been suggested that the observations of the KL nebula require the presence of large grains (Rouan and Léger, 1984; Knacke and McCorkle, 1987). On the other hand the high degree of polarization observed (up to $40 \%$ at $3.8 \mu \mathrm{m}$; Werner et al., 1983) implies that the scattering grains cannot be that much larger than in the diffuse interstellar medium (i.e., Rayleigh scattering; $a \lesssim 0.5 \lambda / 2 \pi \simeq 3000 \AA$ ).

Generally, protostars are surrounded by an asymmetric density distribution and stellar photons escape more easily in the polar directions than along the equatorial plane. These photons can be reflected off nearby dense clumps or the inside of the cavity blown by the stellar wind. For low optical depths, the intensity of the reflected light is proportional to the scattering optical depth of the reflection nebula, while for high optical depths, it reflects the albedo. A simple theoretical model for an optically thick, isotropically scattering nebula provides a good fit to the observations 

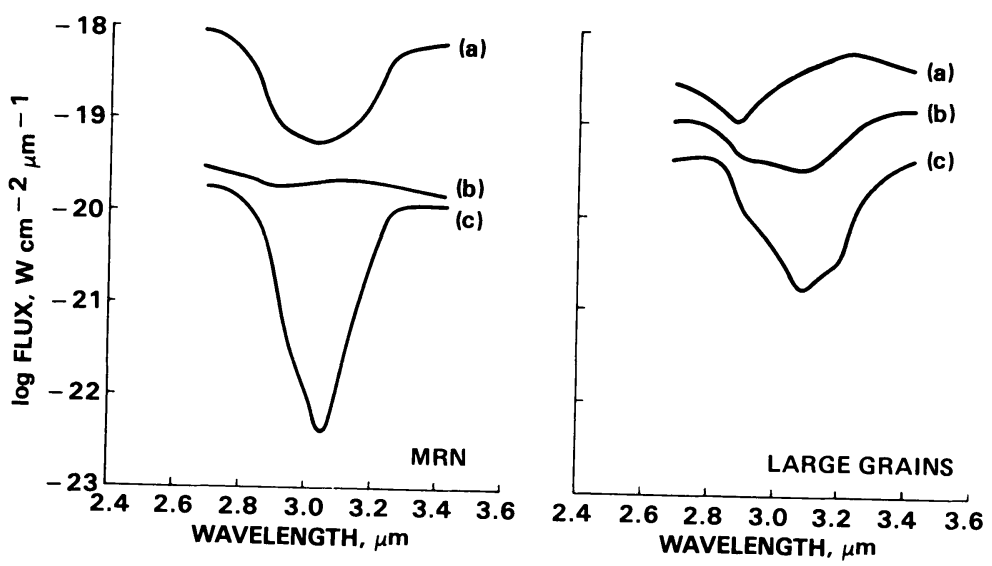

Fig. 2. The shape of the $3.08 \mu \mathrm{m}$ ice band in the scattered light from a reflection nebula for the MRN and a large grain $(500 \leq a(\AA) \leq 5000)$ dust model. For the MRN model the albedo shows a pronounced ice band (curve a: optically thick nebula), while the ice mantles have little influence on the scattering cross sections (curve b: optically thin nebula). Curve $c$ shows the effect of adding foreground extinction. For large grains, the albedo (curve a) and the scattering cross section (not shown) are very similar. Here curves $b$ and $c$ show the effects of adding varying amounts of foreground extinction to curve a.

of the well-studied OMC 2-IRS 1 nebula (Fig. 1 ; Pendleton et al., 1989). Note that the decrease to short wavelengths in this model is due to the assumed, low temperature of the illuminating source. The well-known MRN model for the dust in the diffuse interstellar medium has been used in these calculations (Mathis et $a l ., 1977$; Draine and Lee, 1984). Thin ice mantles $(\Delta a=30 \AA)$ have been added to this grain model, but their only influence is to produce a small $3.08 \mu \mathrm{m}$ ice band. Thus, these photometric observations do not necessarily require modification of interstellar grain models, although it should be emphasized that models based upon larger than average grains can also provide good fits to the observations. The observed spatial distribution of the scattered light is more difficult to reproduce with the MRN model. Furthermore, the degree of polarization is relatively low $(\simeq 15 \%$ at $3.8 \mu \mathrm{m})$ in this nebula and, more importantly, it decreases to shorter wavelengths. This is also inconsistent with the MRN model and somewhat larger grains $(a \simeq 5000 \AA)$ are indicated (Pendleton et al., 1989).

Besides polarization, one way to distinguish between large and small grains in reflection nebulae is by studying the shape of the $3.08 \mu \mathrm{m}$ ice band in the reflected light (Knacke and McCorkle, 1987; Pendleton et al., 1989). The energy distribution produced by scattering off large ice grains will show structure around $2.95 \mu \mathrm{m}$, due to the reduced scattering cross section of icy grains at those wavelengths. As a result, the shape of the $3.08 \mu \mathrm{m}$ ice band is quite sensitive to the grain size. This is illustrated in Fig. 2. Of course, extinction by foreground ice grains can partially obscure these differences and the only observable effect may be a wing 
around $2.95 \mu \mathrm{m}$. Such structure has been observed in several protostellar sources including $\mathrm{BN}$ and has originally been interpreted in terms of $\mathrm{NH}_{3}$ molecules mixed into the ice mantle which can also give rise to an (absorption) feature around this wavelength (Knacke et al., 1982). However, a recent study of the ice band in the OMC 1 nebula shows that this structure is indeed due to scattering rather than absorption effects (Knacke and McCorkle, 1987). While the $2.95 \mu \mathrm{m}$ wing is obvious in the BN spectrum obtained with a $22^{\prime \prime}$ beam, its strength is greatly reduced in the $5^{\prime \prime}$ beam and it seems that a large contribution of scattered light in the large beam observations has confused the earlier interpretation. A good fit to the $22^{\prime \prime}$ observations can be obtained by including the effects of scattering by large ice grains in the reflection nebula surrounding BN $(a \approx 5000 \AA$; Pendleton et $a l ., 1989)$. Thus, this provides the first unequivocal proof that large ice grains are indeed required to explain the observations of this reflection nebula. It should also be emphasized that the icy grains providing most of the ice band extinction along the line of sight towards BN are much smaller (Hagen et al., 1983). This increase in the local grain size may well result from the high density $\left(n \gtrsim 10^{8} \mathrm{~cm}^{-3}\right)$ in the BN-KL reflection nebula as compared to the surrounding quiescent molecular cloud $\left(n \lesssim 10^{5} \mathrm{~cm}^{-3}\right.$ ) and the resulting increase in the coagulation rate (cf., §5). In any case, spectroscopic and polarimetric studies of IR reflection nebulae can provide a good handle on the sizes of the scattering dust.

\section{ICY GRAIN MANTLES}

\subsection{GRAIN SURFACE CHEMISTRY}

Gas phase species colliding with cold $(\approx 10 \mathrm{~K})$, interstellar grains are expected to stick to the surface with almost unit probability (cf., the review by Tielens and Allamandola, 1987b). Although the flux of incoming species is typically very low (few/day), all heavy gas phase species should have accreted in about $10^{5}$ yrs at a density of $10^{4} \mathrm{~cm}^{-3}$. Since some of the accreted species will be very mobile (i. e., atomic $H$ and $O$ ) on such surfaces, reactions between them as well as with immobile species are important and will lead to the formation of a molecular grain mantle whose composition may be quite different from that of the gas phase (Tielens and Hagen, 1982; Tielens, 1983; d'Hendecourt et al., 1985). This is illustrated in Fig. 3 which compares the calculated gas phase and grain mantle composition of interstellar clouds as a function of total gas phase density.

In the gas phase, hydrogen is mainly in the form of $\mathrm{H}_{2}$, carbon in the form of $C O$, nitrogen and the remainder of the oxygen are either in atomic $(N, O)$ or diatomic $\left(\mathrm{N}_{2}, \mathrm{O}_{2}\right)$ form, depending on the physical conditions (cf., Fig. 3). At low densities atomic $H$ is an important constituent of the gas phase and it dominates the grain surface reactions. Under such reducing conditions, hydrides such as $\mathrm{H}_{2} \mathrm{O}$, and $\mathrm{H}_{2} \mathrm{CO}$ formed from $\mathrm{O}, \mathrm{O}_{2}$, and $\mathrm{CO}$ are calculated to be abundant grain mantle species (cf., Fig. 3). Note that the grain mantle abundance of $\mathrm{NH}_{3}$ is low since most of the elemental $N$ in the gas phase is in the form of $N_{2}$ and its reaction with $H$ is inhibited by a large activation barrier. In these calculations, reduction of $C O$ was stopped at $\mathrm{H}_{2} \mathrm{CO}$. Recent laboratory experiments suggest that the $\mathrm{H}$ addition to $\mathrm{H}_{2} \mathrm{CO}$ has a low activation energy (Zhao, private communication) and most of the $\mathrm{H}_{2} \mathrm{CO}$ in the grain mantles might actually be reduced to $\mathrm{CH}_{3} \mathrm{OH}$. 

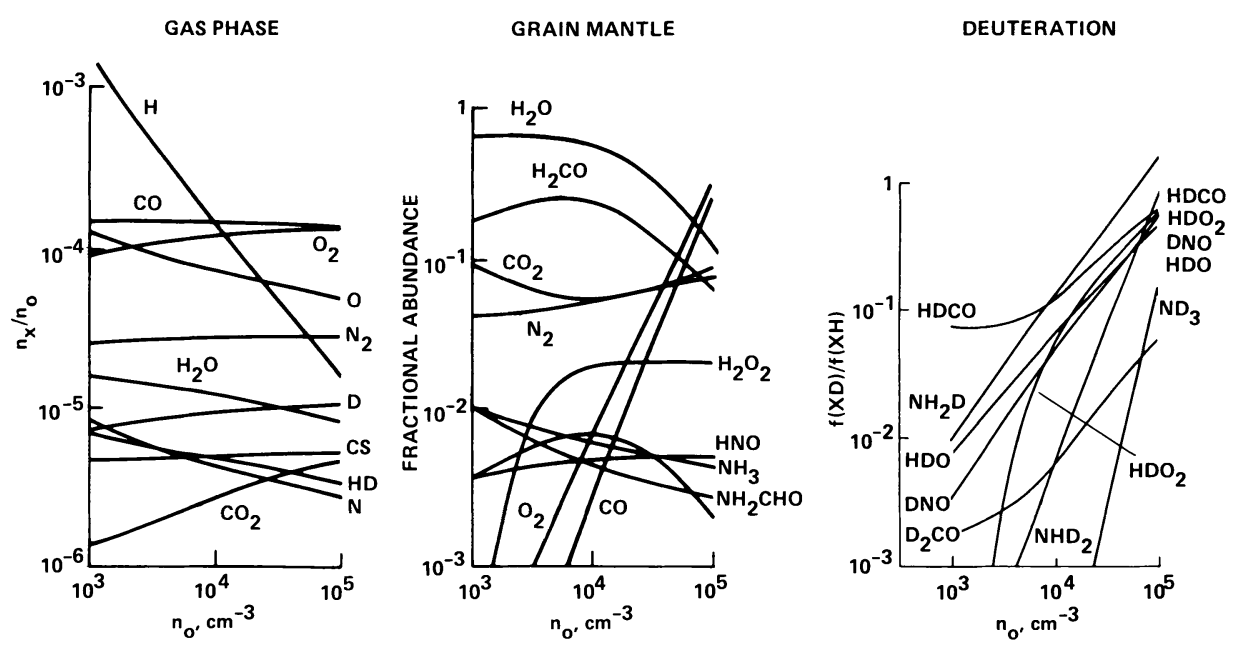

Fig. 3. A comparison of the calculated gas phase and solid state composition of a molecular cloud. When atomic $H$ dominates over heavy species in the gas phase, accreting species will be hydrogenated. Otherwise grain mantles will reflect the gas phase more directly. Note that large deuterium enrichments are expected to be a characteristic of grain mantles as a result of the high atomic $D / H$ ratio in the gas phase (Tielens, 1983).

At high densities, the gas phase abundance of heavy species is much larger than that of atomic $H$ and as a result the composition of the grain mantle will be quite different. At low, gas phase, atomic $O$ abundances few grain surface reactions will occur and an inert grain mantle will result, consisting mainly of $\mathrm{CO}$ and $\mathrm{O}_{2}$ (cf., Fig. 3), which reflects their gas phase dominance. However, if the gas phase $\mathrm{O}$ abundance is high, $\mathrm{CO}$ may be oxidized to $\mathrm{CO}_{2}$. It has been suggested that this reaction is inhibited by an activation barrier (Grim and d'Hendecourt, 1986). However, although $O$ atoms can be stored in a $C O$ matrix, immediately upon warmup $C_{2}$ is formed (Tielens and Hagen; unpublished). The reaction seems therefore to be mainly inhibited by steric factors rather than an activation barrier and this is expected to be of much less importance on a grain surface than in a matrix.

Grain surface reactions can lead to large deuteration effects (Fig. 3 ; Tielens, 1983). The $D$ and $H$ grain surface reaction networks are very similar and this deuteration reflects directly the high gas phase $D$ to $H$ ratio $\left(\gtrsim 10^{-2}\right)$ which is much larger than the elemental abundance ratio $\left(\approx 10^{-5}\right)$. This is a direct consequence of ion-molecule gas phase chemistry which lead to high abundances of deuterated ions (i.e., $D C O^{+}$), which dissociatively recombine with electrons to form atomic $D$ (Tielens, 1983; Dalgarno and Lepp, 1986).

In summary, theoretical calculations predict the possible presence of three different types of icy grain mantles in molecular clouds depending on the physical 
conditions in the gas phase: 1) a reduced component (i. e., $\mathrm{H}_{2} \mathrm{O}$ and $\mathrm{CH}_{3} \mathrm{OH}$ ); 2) an inert component (i. e., $\mathrm{CO}$ and $\mathrm{O}_{2}$ ); and 3) an oxidized component (i.e., $\mathrm{CO}_{2}$ ). Another characteristic of grain surface reactions is large deuterium fractionation of hydrides. Finally, it should be emphasized that the grain mantle composition is largely determined by the gas phase composition of molecular clouds, which sets the stage for the diffusion limited grain surface reactions. In recent years, the rates of several key reactions have undergone major revisions and their influence on the expected grain mantle composition has not yet been assessed.

\subsection{IR SPECTROSCOPY OF ICY GRAIN MANTLES}

Many objects embedded in or located behind a dense molecular cloud show deep absorption features at $3.08,4.67,6.0,6.85$, and $10 \mu \mathrm{m}$ in their spectrum. Fig. 4 shows the composite IR spectrum of one of the best studied objects, W33A. With the exception of the $10 \mu \mathrm{m}$ band, these features are generally attributed to simple molecules embedded in icy grain mantles along the line of sight. The proposed identifications for these absorption features shown in Fig. 4 have recently been reviewed by Tielens and Allamandola (1987a) and only some new developments will be discussed here.

The strong band at $3.08 \mu \mathrm{m}$ is due to the $\mathrm{OH}$ stretching vibration of $\mathrm{H}_{2} \mathrm{O}$ ice or mixtures dominated by $\mathrm{H}_{2} \mathrm{O}$. Recent studies have shown that the detailed shape of this band is severely influenced by scattered light (Knacke and McCorkle, 1987; cf., §2). This is particularly true for the $2.97 \mu \mathrm{m}$ band, which was previously ascribed to $\mathrm{NH}_{3}$ (Knacke et al., 1982; Tielens and Hagen, 1982), but which is now thought to result (mainly) from scattering by large ice grains.

The $3.08 \mu \mathrm{m}$ ice band shows a low frequency wing, whose origin is unclear. It is probably not due to scattering by large particles (Merrill et al., 1976) as evidenced by the polarization study of BN (cf., Hagen et al., 1983). This wing has also been attributed to absorption by $\mathrm{H}_{2} \mathrm{O}$ hydrogen-bonded to strong bases such as $\mathrm{NH}_{3}$ (Knacke et al., 1982; Hagen et al., 1983), but the upper limit on the $\mathrm{NH}_{3}$ abundance itself is quite small and it cannot be responsible (Tielens and Hagen, 1982). Alternatively, this wing may be caused by overlapping $C H$ stretching absorption features due to organic molecules mixed in with the ice. Indeed, recent higher resolution studies of W33A as well as other protostars have revealed substructure at about $3.5 \mu \mathrm{m}$ which is well matched in detail with the $C H$ stretching vibration of $\mathrm{CH}_{3} \mathrm{OH}$ (Fig. 5; Baas et al., 1989). More substructure is present in these spectra, perhaps indicating many blended $C H$ stretching modes in a family of organic compounds. However, this view is not generally accepted (cf., Baas et al., 1989). Further high resolution observations of a larger sample of objects will be very important to resolve this issue.

The 4 to $5 \mu \mathrm{m}$ region is very characteristic for triple bond and cumulative double bond vibrations. W33A shows a strong band at $4.62 \mu \mathrm{m}$, which has been assigned to the $C \equiv N$ stretching mode in isonitriles or nitriles close to electronegative groups (d'Hendecourt et al., 1986; Larson et al., 1985). A direct comparison with the laboratory spectrum of a specific candidate molecule has not yet been made. Laboratory UV photolysis studies of simple molecular mixtures containing carbon, nitrogen, and oxygen often show a strong absorption feature at this wavelength which matches the observed interstellar feature very well (Lacy et al., 1984). The carrier of this feature is not well known, although identification with a nitrile seems 


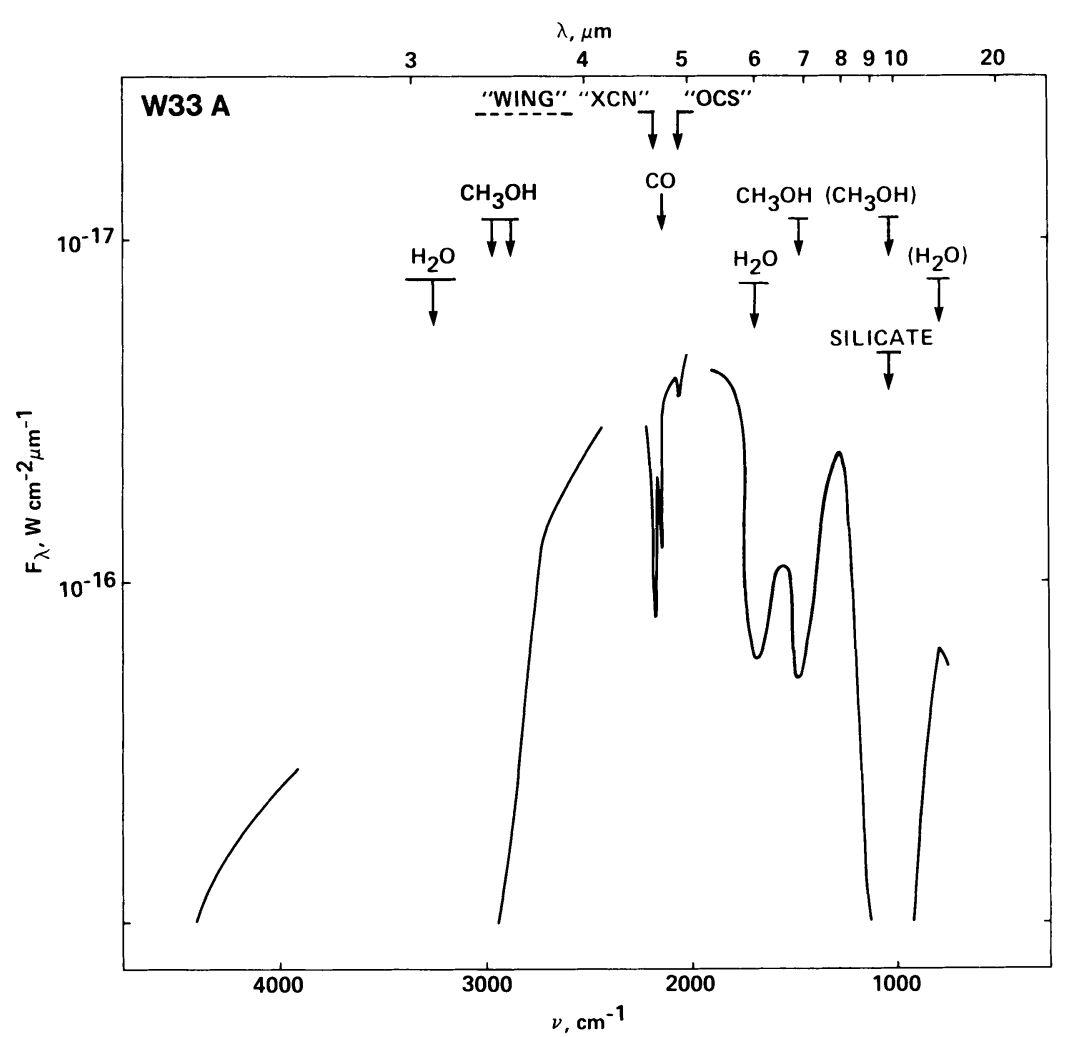

Fig. 4. The IR spectrum of the protostar W33A. Identifications for the observed absorption features in terms of simple "icy" molecules are indicated on the top. Some of these are, however, not unequivocally accepted. The deep $10 \mu \mathrm{m}$ band is generally attributed to refractory silicates. See text for details.

reasonable (d'Hendecourt et al., 1986). It has been suggested that the laboratory feature, and by inference the interstellar feature, is actually due to the $O C N^{-}$ion (Grim and Greenberg, 1987). In salts this ion shows an absorption feature at about this wavelength, although the exact peak position depends strongly on the cation present. No positive evidence for the presence of this anion in the laboratory studies has, however, been presented. Moreover, the peak frequency of an absorption feature due to an ion will be very sensitive to the local matrix environment (i. e., cation, degree of solvation). Yet, the measured laboratory peak frequency does not shift upon warm up, $\mathrm{H}_{2} \mathrm{O}$ ice evaporation and organic residue formation. Clearly, the carrier of this laboratory band cannot be an ion and further laboratory studies are required to identify it. Finally, it has been suggested that the interstellar $4.62 \mu \mathrm{m}$ band is actually due to the $\mathrm{SiH}$ stretching vibration in organic-silicon compounds (Nuth and Moore, 1988). Such molecular structures may result from UV photolysis of icy molecules containing silicon compounds (e.g., $\mathrm{SiH}_{4}$ ). A detailed comparison of these laboratory spectra with the observations could be very interesting. 
BAAS et al. (1988)

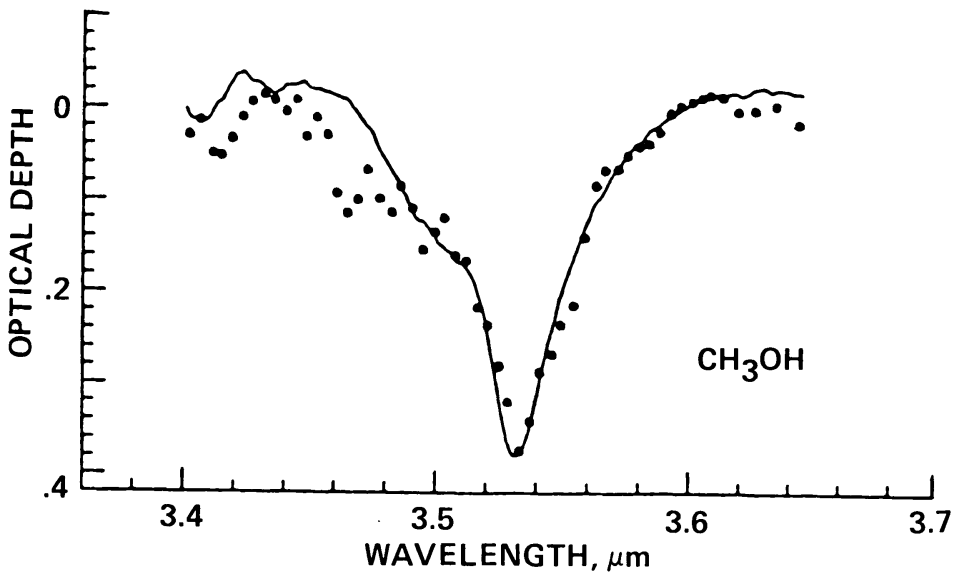

Fig. 5. A weak feature at about $3.5 \mu \mathrm{m}$ has been detected in the spectrum of W33A (dots; Baas et al., 1989). It seems to be present in the spectra of other sources as well. A good match to the shape of this feature is obtained with solid $\mathrm{CH}_{3} \mathrm{OH}$ (solid line).

Many sources show a strong, narrow absorption feature near $2140 \mathrm{~cm}^{-1}(4.67$ $\mu \mathrm{m}$ ), which is commonly attributed to solid $C O$ (Lacy et al., 1984; Geballe, 1986). Fig. 6 shows a few, high resolution $(\lambda / \Delta \lambda \approx 1500)$ spectra obtained in this wavelength region with the CGAS spectrometer at the IRTF (Tielens et al., 1989). Because of selection rules, absorption due to solid and gas phase $C O$ are readily separated in these spectra (cf., Allamandola, 1984). At this resolution the interstellar, solid $C O$ line, which has a typical linewidth of about $0.01 \mu \mathrm{m}$, is resolved and other emission components (ie., hot gas phase $C O$ and $H \mathrm{I}$ ) can be readily separated. This allows, for the first time, a detailed comparison with laboratory spectra. Note that cold molecular cloud material produces, at most, $3 \%$ absorption features at this resolution. Most observed spectra show a narrow $(0.01 \mu \mathrm{m})$ component at $\approx 4.67 \mu \mathrm{m}$. Sometimes, a long wavelength wing, probably due to a broad $(\approx 0.02$ $\mu \mathrm{m})$, weak, underlying component $(\approx 4.68 \mu \mathrm{m})$, is also present (cf., NGC 7538 IRS 9 ). In a few cases an increased width or shifted peak position indicates a different $\operatorname{mix}$ (cf., GL 961).

Laboratory studies show that the peak position and shape of the fundamental vibration of $C O$ mixed into cold, molecular ices is a complex function of its interaction with the surrounding matrix (Sandford et al., 1988). Among the possible effects are electrostatic dipole-induced dipole interaction as for $\mathrm{H}_{2} \mathrm{O} / \mathrm{CO}$ complexes (Hagen and Tielens, 1981), the presence of substitutional as well as interstitial sites as in disordered amorphous ices (Sandford et al., 1988), and electron donation by surrounding strong bases (cf., Hollim and Pritchard, 1980). The absorption feature due to $\mathrm{CO}$ molecules isolated in a matrix dominated by $\mathrm{H}_{2} \mathrm{O}$ occurs at about 4.68 $\mu \mathrm{m}$ and has a width of about $0.02 \mu \mathrm{m}$. Pure solid $C O$ absorbs at about $4.675 \mu \mathrm{m}$ with a width of about $0.005 \mu \mathrm{m}$. Doping of the $C O$ matrix with traces of other species leads to a broadening but little shift of this line. Since the observed 3.08 

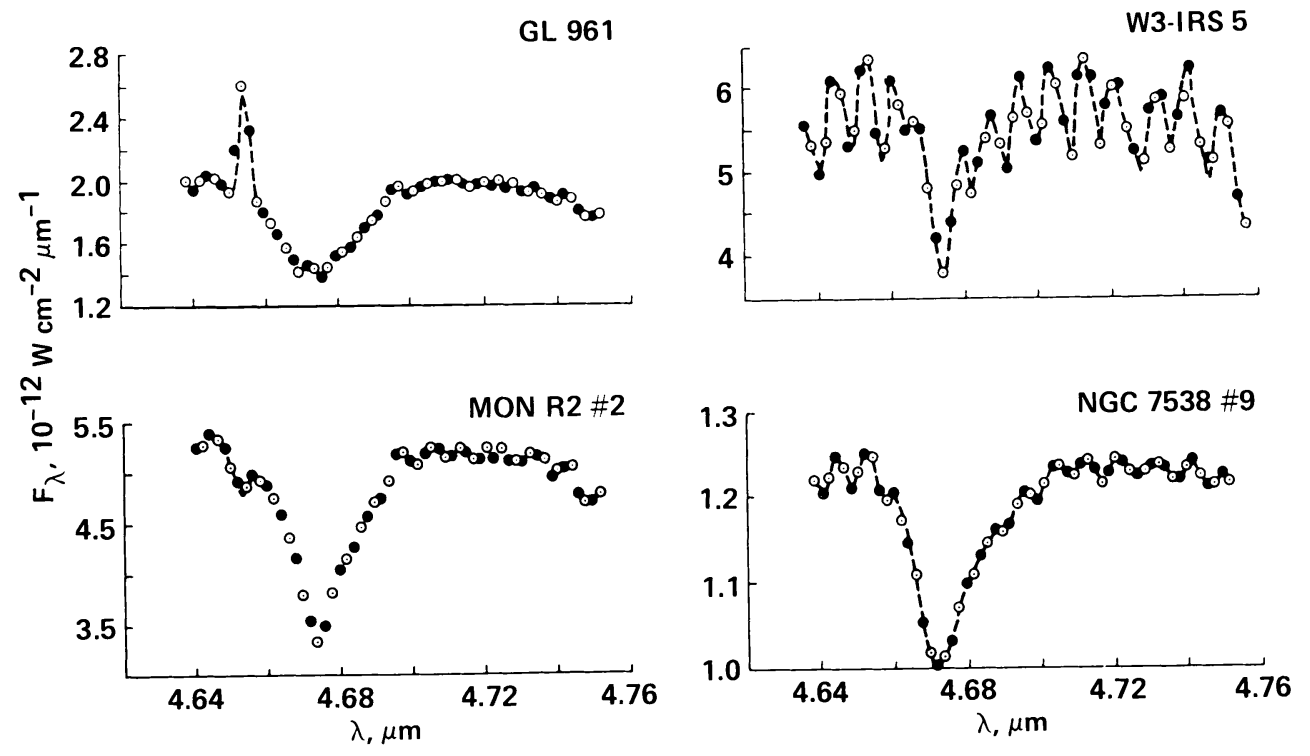

Fig. 6. Some recent high resolution spectra of protostars in the solid $C O$ region (Tielens et al., 1989). Besides the solid $C O$ band at about $4.67 \mu \mathrm{m}$, these spectra often show broad $(\Delta v \approx 50$ $\left.\mathrm{km} \mathrm{s}^{-1}\right)$, hot $(T \approx 1000 \mathrm{~K})$ gas phase $C O$ lines, possibly in a circumstellar disk. The HI 7-5 recombination line is also often present. Open and closed symbols indicate separate grating settings. Dashed lines have been drawn to guide the eyes. The variations in the shape of the solid $C O$ band (i.e., peak position and width) are probably due to variations in the composition of interstellar icy grain mantles.

$\mu \mathrm{m}$ ice band attest to the presence of $\mathrm{H}_{2} \mathrm{O}$ dominated ice mantles, it is tempting to attribute the observed, relatively broad, underlying $\mathrm{CO}$ component to $\mathrm{CO}$ in $\mathrm{H}_{2} \mathrm{O}$ dominated grain mantles, while the narrow $C O$ component may be due to more pure $\mathrm{CO}$ ices containing little $\mathrm{H}_{2} \mathrm{O}$. Indeed, a good fit to the narrow interstellar component can be obtained with pure $C O$ (cf., Fig. 7). Note that, although some other non-polar mixtures can also provide reasonable agreement, these high resolution studies show that contrary to what was previously suggested (Sandford et al., 1988), mixtures dominated by $\mathrm{CO}_{2}$ do not. In any case, these $\mathrm{CO}$ observations do reveal, for the first time, the presence of at least two distinct grain mantle components in interstellar clouds, often along the same line of sight.

The 5-8 $\mu \mathrm{m}$ region is characteristic for $C H, N H$ and $O H$ bending and deformation modes and the strong $C=O$ stretching mode. Two absorption features (at 6.0 and $6.85 \mu \mathrm{m}$ ) have been detected in this wavelength region in protostellar objects. These have been attributed to the $\mathrm{OH}$ bending mode in $\mathrm{H}_{2} \mathrm{O}$ and the $\mathrm{CH}$ deformation mode in $\mathrm{CH}_{3} \mathrm{OH}$ (Tielens et al., 1984). Some low resolution $(\lambda / \Delta \lambda \approx 50)$, interstellar spectra have shown evidence for spectral structure in this wavelength region, which has been interpreted in terms of carbonyl bearing molecules such as $\mathrm{H}_{2} \mathrm{CO}$ (Tielens and Allamandola, 1987a).

Recent moderate resolution $(\lambda / \Delta \lambda \approx 150), 5-8 \mu \mathrm{m}$ spectra obtained with the 


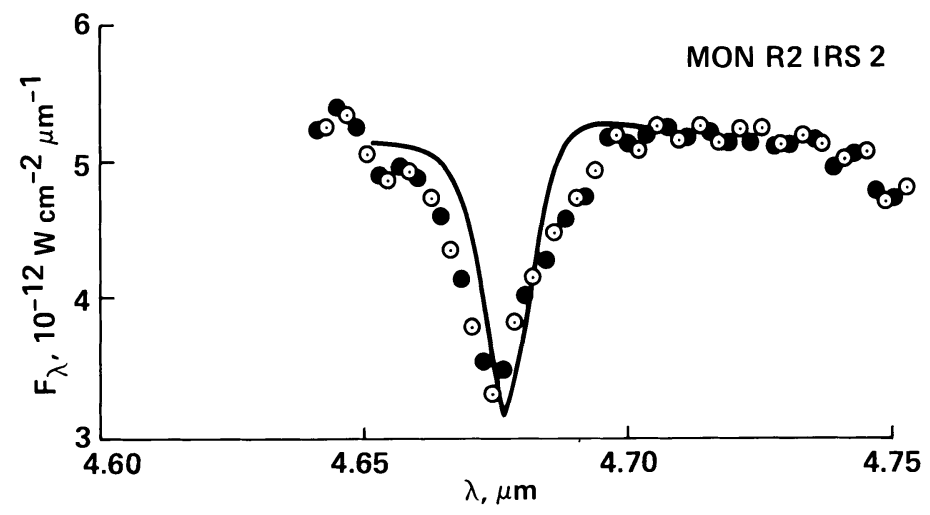

Fig. T. Observations toward Mon R2-IRS 2 compared to the laboratory spectrum of a $C O$ dominated mixture $\left(\mathrm{CO}: \mathrm{H}_{2} \mathrm{O}=10: 1\right)$, demonstrating the good fit possible with non-polar mixtures. Open and closed symbols represent separate grating settings. The mismatch in peak position is within the uncertainty. Note that the solid $\mathrm{CO}$ band in $\mathrm{H}_{2} \mathrm{O}$ dominated mixtures absorbs at longer wavelengths and has twice the observed interstellar width. A hint of a component with these characteristics seems to be present in the observed spectrum.
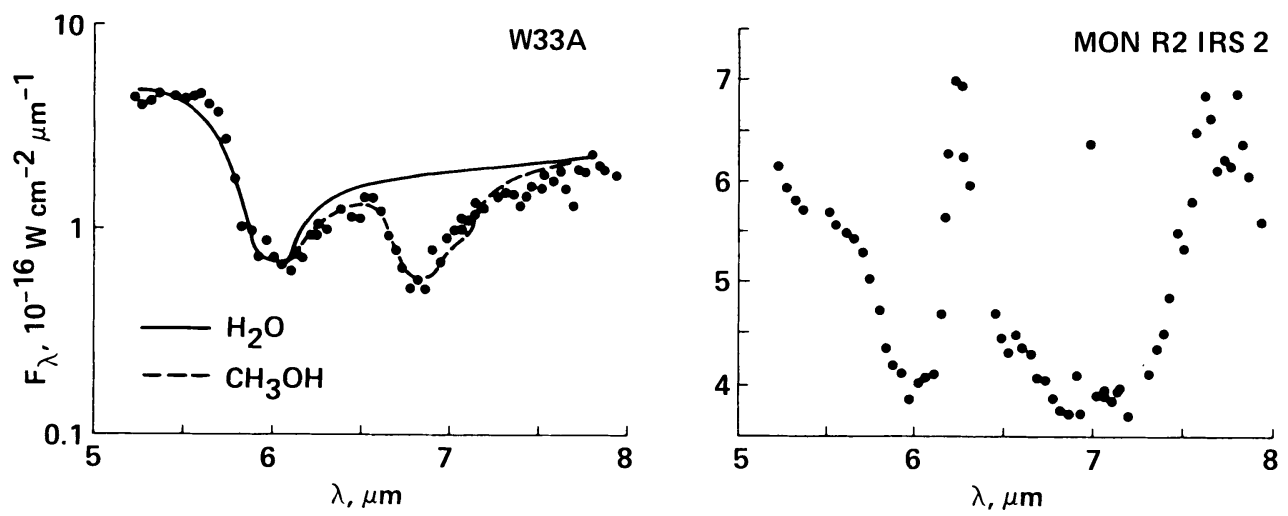

Fig. 8. Recent 5-8 $\mu \mathrm{m}$ spectra of two protostars. W33A shows absorption features at 6.0 and $6.85 \mu \mathrm{m}$ which are particularly well matched by the laboratory spectra of $\mathrm{H}_{2} \mathrm{O}$ and $\mathrm{CH}_{3} \mathrm{OH}$ added to the $\mathrm{H}_{2} \mathrm{O}$ absorption. Although a weak $6.0 \mu \mathrm{m}$ absorption band may be present, the spectrum of Mon R2 IRS 2 is dominated by the well known 6.2 and $7.7 \mu \mathrm{m}$ emission features and the ArII and Pf $\alpha$ emission lines. Recognition of these emission components in previous studies was hampered by insufficient spectral resolution. 
KAO are in good agreement with previous lower resolution spectra (Fig. 8). The 6.0 and $6.85 \mu \mathrm{m}$ feature in the spectrum of W33A are indeed fitted very well by laboratory spectra of $\mathrm{H}_{2} \mathrm{O}$ and $\mathrm{CH}_{3} \mathrm{OH}$ (Fig. 8). The latter identification is however not unequivocally accepted (see below). Carbonyl bearing molecules (eg., $\mathrm{H}_{2} \mathrm{CO}$ ) show a strong $C=O$ stretching vibration at about $5.8 \mu \mathrm{m}$. The smoothness of the $6.0 \mu \mathrm{m}$ feature in W33A implies an upper limit of about $0.2 \%$ on the abundance of $\mathrm{C}=\mathrm{O}$ groups with respect to $\mathrm{H}_{2} \mathrm{O}$. The spectrum of Mon R2-IRS 2 is strikingly different from that of W33A. However, rather than the presence of carbonyl groups as previously suggested (Tielens et al., 1984), these higher resolution observations show that this is due to the presence of strong PAH emission features at 6.2, 7.7, and probably $5.2 \mu \mathrm{m}$ (cf., Bregman, 1989). Note that the $7.7 \mu \mathrm{m}$ feature in this source is particularly well resolved into features at about 7.5 and $7.8 \mu \mathrm{m}$. The $A r I I$ line at $6.99 \mu \mathrm{m}$ is also obvious at this resolution. These emission features probably arise in the nearby compact HII region associated with Mon R2-IRS 1. Although a narrow $6.0 \mu \mathrm{m}$ absorption band similar to that in W33A seems to be present in this source, there is little evidence for a $6.85 \mu \mathrm{m}$ absorption band. Thus, presently, there is no direct evidence for complex molecular grain mantles in the 5-8 $\mu \mathrm{m}$ region of the spectrum. Indeed, the profiles of the 6.0 and $6.85 \mu \mathrm{m}$ band in W33A, NGC 7538-IRS 9, W3 IRS 5, and AFGL 2136, are very similar, despite the large difference in optical depth (cf., Tielens and Allamandola, 1987a).

\subsection{GRAIN MANTLE ABUNDANCES}

Table 1 summarizes typical abundances for several interesting molecules in icy grain mantles relative to $\mathrm{H}_{2} \mathrm{O}$. This molecule has always the largest column density along a line of sight. However, as discussed above, the solid $C O$ data implies the existence of at least two, distinctly different grain mantle components along many lines of sight. Typical estimates for the solid $C O$ in each of these components are listed separately. Although many questions remain, a suggestive comparison can be made between observed and calculated abundances (cf., Table 1). For example, several of the molecules predicted to be present have been observed with approximately the right abundance. The detection of grain mantles dominated by non-polar molecules rather than $\mathrm{H}_{2} \mathrm{O}$ is also in good agreement with theoretical predictions. The detection of interstellar oxidized grain mantles probably has to await space-born IR spectrometers.

There is some controversy on the abundance of $\mathrm{CH}_{3} \mathrm{OH}$. The abundance, which has been inferred from the $3.5 \mu \mathrm{m}$ band, is much lower than that obtained from the $6.85 \mu \mathrm{m}$ band and may imply that the latter is largely due to molecules other than $\mathrm{CH}_{3} \mathrm{OH}$ (Baas et al., 1989). However, part of this discrepancy may actually reflect the uncertainty in the underlying continuum of the low frequency wing on the $3.08 \mu \mathrm{m}$ ice band, particularly if this wing is due to blending of overlapping $C H$ stretching bands (cf., §3.2).

Perhaps not surprising, the Leiden group has recently suggested that, like their assignment of the $4.62 \mu \mathrm{m}$ band, the $6.85 \mu \mathrm{m}$ band is also due to an ion produced by UV photolysis (i.e., $\mathrm{R}-\mathrm{NH}_{3}^{+}$or $\mathrm{NH}_{4}^{+}$; Schutte, 1988; Grim, 1988). Laboratory UV photolysis studies on $\mathrm{NH}_{3} / \mathrm{O}_{2}$ mixtures demonstrate that a simple nitrogen and/or oxygen containing compound produces an absorption feature around 6.7 $\mu \mathrm{m}$. A similar band appears upon UV photolysis of a $\mathrm{H}_{2} \mathrm{O} / \mathrm{NH}_{3} / \mathrm{CO}_{2} \mathrm{O}_{2}(10 / 1 / 1 / 1)$ mixture, which by inference is then attributed to the same compound. This band 
TABLE 1: COMPOSITION OF INTERSTELLAR ICY GRAIN MANTLES

\begin{tabular}{|c|c|c|c|c|}
\hline \multirow[b]{2}{*}{ Species } & \multicolumn{2}{|c|}{ Absorption features } & \multicolumn{2}{|c|}{ Abundance $^{1}$} \\
\hline & $\lambda[\mu \mathrm{m}]$ & $\nu\left[\mathrm{cm}^{-1}\right]$ & Observed & Calculated $^{2}$ \\
\hline \multirow[t]{2}{*}{$\mathrm{H}_{2} \mathrm{O}$} & 3.08 & 3250 & - & - \\
\hline & 6.00 & 1650 & 100 & 100 \\
\hline \multirow[t]{2}{*}{$C O^{3}$} & 4.68 & 2135 & $0-5$ & 0.4 \\
\hline & 4.67 & 2140 & $0-25$ & - \\
\hline \multirow[t]{2}{*}{$\mathrm{CH}_{3} \mathrm{OH}$} & 3.5 & 2830 & $7^{4}$ & - \\
\hline & 6.85 & 1460 & 50 & $(40)^{5}$ \\
\hline$X C N$ & 4.61 & 2167 & $(4)^{6}$ & - \\
\hline$O C S$ & 4.90 & 2040 & $(0.05)^{7}$ & 0.4 \\
\hline \multirow[t]{2}{*}{$\mathrm{NH}_{3}$} & 2.95 & 3375 & $<5$ & 1 \\
\hline & 6.10 & 1625 & - & - \\
\hline $\mathrm{H}_{2} \mathrm{~S}$ & 3.94 & 2540 & 0.3 & 0.03 \\
\hline $\mathrm{CH}_{4}$ & 7.70 & 1300 & $<1$ & 0.007 \\
\hline \multirow[t]{2}{*}{$\mathrm{H}_{2} \mathrm{CO}$} & 3.53 & 2835 & $<0.2$ & $(0)^{5}$ \\
\hline & 5.80 & 1720 & - & - \\
\hline $\mathrm{CO}_{2}$ & 4.28 & 2337 & -8 & 9 \\
\hline
\end{tabular}

${ }_{1}$ Abundances of molecules in grain mantles relative to $\mathrm{H}_{2} \mathrm{O}=100$. ${ }^{2}$ Calculated abundances for a reducing atmosphere $\left(n_{o}=\right.$ $10^{4} \mathrm{~cm}^{-3}$; Tielens and Hagen, 1982).

${ }^{3}$ The 4.68 and $4.67 \mu \mathrm{m}$ bands refer to the two independent $C O$ components observed. Both abundance estimates are relative to the total $\mathrm{H}_{2} \mathrm{O}$ (ice) column density along the line of sight.

${ }^{4}$ The observed abundance of $\mathrm{CH}_{3} \mathrm{OH}$ is controversial (see text).

${ }^{5}$ Assuming all $\mathrm{H}_{2} \mathrm{CO}$ converted into $\mathrm{CH}_{3} \mathrm{OH}$ by grain surface reactions.

${ }^{6}$ Thought to result from UV photolysis. Abundance estimated from laboratory studies (d'Hendecourt et al., 1986).

${ }^{7}$ Assuming absorption is due to $O C S$.

${ }^{8}$ Presently unobservable due to telluric $\mathrm{CO}_{2}$. 
shows considerable shift upon warm up, consistent with ion-solvation (or annealing) effects. A good fit to the interstellar band can be obtained with a sample warmed up to $180 \mathrm{~K}$ (Grim, 1988). However, similar processing of other equally relevant interstellar mixtures does not provide such a good agreement (d'Hendecourt, 1984; Schutte, 1988). The temperature sensitivity is also difficult to reconcile with the similarity in the $6.85 \mu \mathrm{m}$ profile observed in a variety of sources. In contrast, the invariance of the shape of the $6.85 \mu \mathrm{m}$ feature is readily understood assuming $\mathrm{CH}_{3} \mathrm{OH}$ absorption. This and the detection of the $3.5 \mu \mathrm{m}$ absorption (see Fig. 5) makes $\mathrm{CH}_{3} \mathrm{OH}$ the preferred interpretation in the author's opinion.

\section{ORGANIC REFRACTORY GRAIN MANTLES}

UV photolysis of simple molecular ices produces reactive radicals (such as $H, O$ and $O H$ ) often in electronic or vibrationally excited states. These radicals can react with their neighbours or diffuse through the ice and react with other species present. Because of the large amount of excess energy available, even reactions with molecules having closed electronic shells, which normally have appreciable activation barriers, become possible. Since these radicals may transfer most of their excess energy through collisions with matrix molecules before finding a reaction partner, they can also be stored at low temperatures in interstitial or substitutional sites. Upon warm-up above $30 \mathrm{~K}$ these radicals can diffuse again and react, forming more complex molecules and resulting in a so-called organic refractory grain mantle (Greenberg, 1979; d'Hendecourt et al., 1982, 1986; Schutte, 1988). Whether this actually takes place inside dense interstellar clouds depends on two conditions: The presence of an internal UV source and the presence of a (transient) heating mechanism for grain mantles (cf., Tielens and Allamandola, 1987b). Cosmic rays may play a major role for both conditions. Cosmic ray excitation of $\mathrm{H}_{2}$ molecules leads to UV fluorescence in the Lyman-Werner bands, resulting in an average internal UV field of about $10^{3}$ photons $\mathrm{cm}^{-2} \mathrm{~s}^{-1}$ (Prasad and Tarafdar, 1983; Sternberg et al., 1987). This is sufficient to produce a stored radical concentration of about $1 \%$ in a grain mantle in about $2 \times 10^{4}$ yrs. A larger concentration of radicals is inhibited by recombination of "hot", diffusing radicals with stored radicals. However, transient heating of grain mantles by cosmic rays to about 30K occurs at a similar timescale (Léger et al., 1985) and will lead to diffusion and reaction of these stored radicals. The process can then start over again. Grain-grain collisions with relative velocities in excess of $50 \mathrm{~m} / \mathrm{s}$ will also lead to warm up above $30 \mathrm{~K}$ and can trigger the diffusion and reaction of stored radicals (d'Hendecourt et al., 1982).

Extensive laboratory studies have been done on UV photolysis and warm-up of molecular ices containing $\mathrm{H}_{2} \mathrm{O}, \mathrm{NH}_{3}, \mathrm{CO}$ and $\mathrm{CH}_{4}$ at various concentrations (d'Hendecourt et al., 1986; Schutte, 1988). Besides the original matrix molecules, many simple newly formed molecules (i.e., $\mathrm{HCOOH}, \mathrm{HCON}_{2}, \mathrm{CO}_{2}, \mathrm{CH}_{3} \mathrm{OH}$, $H N O$ ) are released upon warm-up. These result from reactions between $O, H, O H$ and $\mathrm{NH}_{2}$ radicals and matrix molecules (Schutte, 1988). After warm-up above 150K most of the deposited matrix molecules (i. e., $\mathrm{H}_{2} \mathrm{O}$ ) have evaporated and a refractory residue with an enhanced $C$ abundance is left behind. The composition of some of these residues has been determined by gas chromatography/mass spectrometer analysis (Table 2; Schutte, 1988). Similar molecules are produced by energetic ion bombardment of such ices (cf., Rossler, 1986 and references therein). Typically, these residues consist of small organic molecules with $\mathrm{OH}, \mathrm{C}=\mathrm{O}, \mathrm{NH}_{2}$ and $\mathrm{CH}_{2}$ 
groups and contains 5 to 7 heavy elements of which 2 to 3 are $C$ atoms (Smaller ones evaporate below room temperature). Essentially, these molecules are formed by the addition of a few simple building blocks, such as $\mathrm{OH}, \mathrm{NH}_{2}$, and $\mathrm{HCO}$, and plausible schemes for their chemical formation can be made (Schutte, 1988). Note also that the residue has an enhanced carbon content compared to the original mixture. In view of the limited number of building blocks, pronounced chemical selectivity will result. The residues studied are dominated by carboxylic acids, alcohols, and amides, which merely reflects the dominance of saturated hydrides and $C O$ in the initial mixture. Although a different initial mixture may result in a quite different chemical composition of the residue, we can conclude in general that UV photolysis of icy grain mantles will be chemically specific.

TABLE 2: THE COMPOSITION OF AN ORGANIC RESIDUE ${ }^{1}$

\begin{tabular}{|c|c|c|}
\hline Species & Formula & Abundance \\
\hline Glycolic acid & $\mathrm{HOCH}_{2} \mathrm{COOH}$ & 0.38 \\
\hline Hydroxyacetamide & $\mathrm{HOC} \mathrm{H}_{2} \mathrm{CON} \mathrm{H}_{2}$ & 0.13 \\
\hline Oxamic acid & $\mathrm{H}_{2} \mathrm{NCOCOOH}$ & 0.05 \\
\hline Glycerol & $\mathrm{HOCH}_{2} \mathrm{CHOHCH}_{2} \mathrm{OH}$ & 0.06 \\
\hline Oxamide & $\mathrm{H}_{2} \mathrm{NCOCON} \mathrm{H}_{2}$ & 0.15 \\
\hline Glyceric acid & $\mathrm{HOCH}_{2} \mathrm{CHOHCOOH}$ & 0.07 \\
\hline Glyceramide & $\mathrm{HOCH}_{2} \mathrm{CHOHCON} \mathrm{H}$ & 0.16 \\
\hline
\end{tabular}

About $1 \%$ by mass of the original mixture is converted into this residue (Schutte, 1988). Over the lifetime of the cloud as much as $30 \%$ of all the carbon would be locked up in organic refractory grain mantles. This seems somewhat high, since the IR spectra of protostars are dominated by simple ices (cf., §3.2), containing typically only $5 \%$ of the $O$ and perhaps an equal amount of $C$, rather than by organic residues. In particular, presently, only the $4.62 \mu \mathrm{m}$ band is generally attributed to UV photolyzed grain mantles. All other observed absorption features may result from accretion and surface reactions. Absorption features due to organic residues in the $3 \mu \mathrm{m}$ region of interstellar spectra may be "hidden" to some extent by the strong $3 \mu \mathrm{m}$ ice band. However, the 5-8 $\mu \mathrm{m}$ laboratory spectra of such residues also show various strong absorption bands, which have not been observed in interstellar space. Perhaps, the presence of the $6.0 \mu \mathrm{m}$ ice band as well as the low resolution of the observations in this wavelength region have conspired to conceal this complexity. Further spectroscopic studies of protostars are of utmost importance to "directly" link icy grain mantles and organic residues.

Finally, prolonged exposure to UV photons may eventually lead to the loss of most of the elements other than $C$. Indeed, there is some laboratory evidence for the formation of unsaturated carbon bonds upon prolonged photolysis. Eventually, such processing may lead to an amorphous carbon network, consisting of aromatic units linked by aliphatic chains, similar to that resulting from pyrolysis of terrestrial kerogen. A guestimate for the timescale involved for this process in the diffuse interstellar medium is about $1 \mathrm{Myr}$. Inside dense clouds the UV photon density is 
too low for this to be of importance. Whether this process is of importance on a galactic scale depends then on the ratio of this conversion timescale to the shock destruction timescale for the organic residue in the diffuse interstellar medium. It is quite conceivable that the latter is relatively long ( $\gtrsim 10 \mathrm{Myr}$ ) and thus that amorphous carbon a grain component normally assumed to result from processes around red giants, actually is also formed in the ISM.

\section{GRAIN-GRAIN COLLISIONS}

Grain-grain collisions can have a major influence on the grain size distribution. Fig. 9 summarizes the processes that will play a role at various relative collision velocities and impact pressures for $1000 \AA$ silicate grains. At very high collision velocities a strong shock wave will be driven into each grain compressing them to very high pressures. Upon release the grains will be vaporized. Partial vaporization sets in at about $1 \mathrm{Mbar}$ and complete vaporization occurs at about $6 \mathrm{Mbar}$. For somewhat lower impact velocities, the material will melt upon expansion behind the shock. Below the sound velocity, typically about $5 \times 10^{5} \mathrm{~cm} \mathrm{~s}^{-1}$, a sound wave will transfer the impact signal through the grains. For macroscopic bodies, the material strength is quite low, perhaps $1 \mathrm{kbar}$ for a brittle material such as silicates, much less than the theoretical tensile strength $(\approx 150 \mathrm{kbar})$. This difference is due to the initiation, growth and coalescence of (up to cm-sized) cracks, which locally enhance the applied stresses at their tips manyfold. This process dominates the fracturing of macroscopic bodies in, for example, the asteroid belt or the rings of Saturn. However, at the submicron size scales of interstellar grains the stress enhancement is small and this fracturing process is unimportant. The expected strength of a material is then close to the theoretical tensile strength of a material. Indeed, impact studies of submicron grains on silicate slabs show that cratering does not occur below velocities of about $10^{5} \mathrm{~cm} \mathrm{~s}^{-1}$, corresponding to a peak pressure of about $100 \mathrm{kbar}$. Below that velocity impacting grains will just bounce off without damage. Finally, sticking of two grains will not occur until the velocity drops below a critical velocity, which is of the order of $10^{2} \mathrm{~cm} \mathrm{~s}^{-1}$. Here I will mainly concentrate on grain growth through the latter process.

\subsection{COAGULATION}

The growth of larger bodies through coagulation of smaller units is a common process in, for example, aerosol physics and soot formation. Coagulation has also played a major role in the formation of the planetary and cometary bodies in the early solar nebula. Possibly, it may have been of importance for the size distribution of interstellar grains inside dense clouds. Fig. 10 shows a typical example of the microscopic structure of coagulated grains produced by gas evaporation methods in the laboratory (Iijima, 1987). At this scale the particle has a very open structure, consisting of small $(\approx 1000 \AA)$ spherical grains arranged like pearls on a string. This is very characteristic for coagulation through the addition of individual particles.

Lets consider the physics of a grain-grain collision at low relative velocities. Upon collision the two grains elastically deform at the contact point forming a contact circle. This effect, which was already known to Newton, was studied in great detail by Hertz in the middle parts of the 19th century. The attractive forces, due to van der Waals or chemical forces, across this contact area provide the incentive for 
GRAIN-GRAIN COLLISION: $1000 \AA$ SILICATE GRAINS
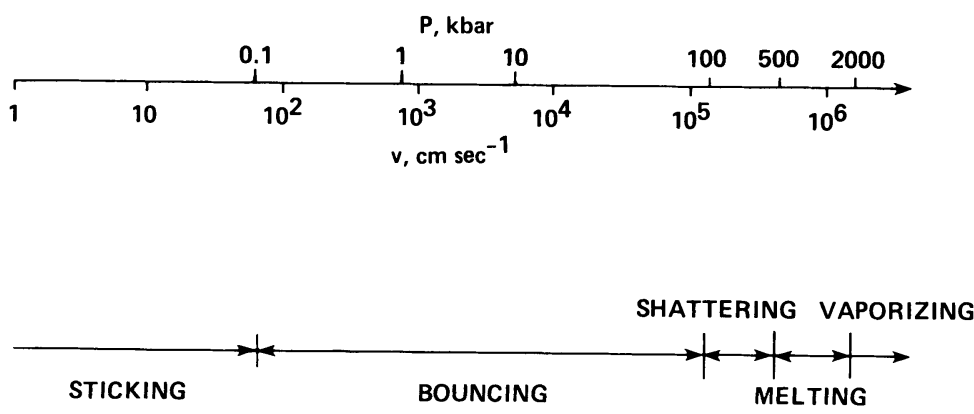

Fig. 9. Depending on the collision velocity, various processes can occur during a grain-grain collision. At low velocities coagulation can be important, while at high velocities the grains will be vaporized. Fragmentation can be important at some intermediate velocities (see text for details). Pressures refer to the shock or sound wave in the solid.

coagulation. During the collision the initial kinetic energy and this attractive energy will be converted into elastic wave energy. Near the contact point these waves will constructively interfere to form a flattened contact area. The elastic energy stored in this deformation will tend to spring the spheres apart, but some of the elastic wave energy will remain behind as internal vibrational (phonon) excitation. Sticking will occur if this is more than the initial kinetic energy. Typically, the internal excitation energy remaining in the grains is comparable to the interaction energy of the two grains (Chokshi et al., 1989). The critical velocity for coagulation, that is the highest velocity for which sticking will still occur, is then given by

$$
v_{c r}=\left(\frac{\gamma}{E R}\right)^{5 / 6} C_{o}
$$

where $\gamma$ is the interface energy, $E$ is Young's modulus, $C_{0}$ is the velocity of sound and $R$ the reduced radius of curvature of the spheres $\left(=R_{1} R_{2} /\left(R_{1}+R_{2}\right)\right)$. Two material parameters are thus important for such calculations: the interface energy and Young's modulus. When the interface energy increases, the area of contact will increase, due to the increased force between the two surfaces. As a result both binding energy and stored elastic energy will increase. However, the former will increase more. Likewise, a smaller Young's modulus (i.e., the material can be elastically deformed more easily) will give rise to a larger contact area. Again the binding energy will increase more than the elastic energy. Thus, while collisions of macroscopic bodies are elastic (Newton 1686), it is well known experimentally that small grains stick easily. This is borne out by our simple model and, essentially, results from the increase of the contact area relative to the grain size with decreasing grain size (Kendall, 1980).

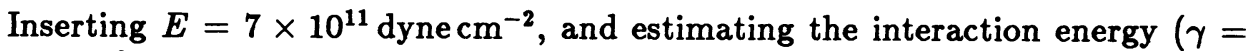

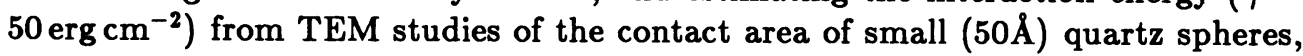




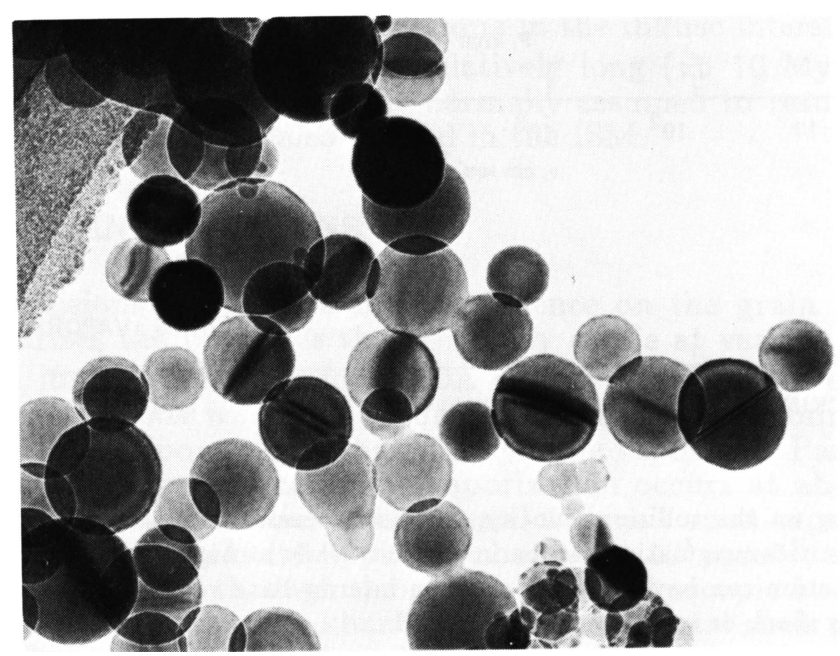

Fig. 10. An electromicrograph showing coagulated spherical $S i$ grains, prepared by a gas evaporation meth d (Iijima, 1987). The dark bands are caused by planar faults. Note the structure of the contact area linking individual grains. The high temperatures in these experiments has allowed plastic deformation of the smallest grains during their agglomeration process. A typical grain has a diameter of $700 \AA$.

leads then to a critical velocity of about $10^{2} \mathrm{~cm} \mathrm{~s}^{-1}$ for $1000 \AA$ silicate grains (Chokshi et al., 1989). Carbonaceous grains have a similar critical velocity. Icy grains stick much better $\left(v_{c r} \approx 2 \times 10^{3} \mathrm{~cm} \mathrm{~s}^{-1}\right)$, since they deform much more easily and bind more strongly (i.e., hydrogen bonding). These critical velocities are in reasonable agreement with experimental data. For example, in sooting flames, $1000 \AA$ carbon particles coagulate while the Brownian velocity is of the order of $30 \mathrm{~cm} / \mathrm{s}$. Nevertheless, these critical velocities are much lower than has sometimes been assumed in astrophysical studies (i. e., $v_{c r} \approx 10^{4} \mathrm{~cm} \mathrm{~s}^{-1}$; Scalo, 1977).

The importance of coagulation will depend on the (relative) velocity field of interstellar grains. At $20 \mathrm{~K}$, the Brownian motion of the grains $\left(\approx 2 \times(1000 \AA / a)^{3 / 2}\right.$ $\mathrm{cm} / \mathrm{s}$ ) is always less than the critical velocity for all grain sizes and sticking is ensured. Comparing the coagulation timescale with the free-fall timescale suggests then that coagulation of interstellar grains will be important for densities in excess of

$$
n_{o} \approx 10^{9}\left(\frac{a}{1000 \AA}\right)^{3} \quad \mathrm{~cm}^{-3}
$$

irrespective of the grain material. Thus, although PAHs and small $(\mathrm{a} \lesssim 25 \AA)$ grains will be depleted at densities of about $10^{4} \mathrm{~cm}^{-3}$, large grains require much higher densities.

The coagulation rate can be enhanced by the presence of turbulence, which 
enhances the relative grain velocities, and by the presence of ice mantles which ensures better sticking at these higher velocities. Volk et al. (1980) and Draine (1985) have analyzed relative grain velocities in turbulent clouds with a Kolmogorov spectrum of turbulent eddies. Typically, relative grain velocities of about $7 \times$ $10^{3} \mathrm{~cm} \mathrm{~s}^{-1}$ are expected for $1000 \AA$ grains at a density of $10^{4} \mathrm{~cm}^{-3}$ in molecular clouds and the collision rate is considerably enhanced. The turbulence in molecular clouds may actually have a magneto-hydrodynamic character and this will change the details of the analysis somewhat (Chokshi et al., 1989). However, the essential point is preserved: some grains couple well to the turbulent gas velocities, while others are largely decoupled. Thus, the relative grain velocity spectrum will reflect rather directly the observed velocity spectrum of the gas. That is (cf., Myers, 1987)

$$
v \approx 3 \times 10^{6} \sqrt{n_{0}} \quad \mathrm{~cm} \mathrm{~s}^{-1}
$$

Using this velocity law implies that the grain-grain collision rate is always less than the free-fall timescale. Thus, once the turbulent velocity drops below the critical velocity, coagulation will dominate. For a silicate grain, coagulation will dominate for densities above

$$
n_{0} \approx 10^{9}\left(\frac{a}{1000 \AA}\right)^{5 / 3} \quad \mathrm{~cm}^{-3}
$$

This is still prohibitively long except for the smallest grains. However, for icy grains the corresponding limit is

$$
n_{0} \approx 10^{4}\left(\frac{a}{1000 \AA}\right)^{5 / 3} \quad \mathrm{~cm}^{-3}
$$

Thus, for typical conditions in molecular clouds, large grains will only coagulate if they are ice coated. Therefore, although coating by an accreted ice mantle itself will only lead to a small increase in the grain size $(\Delta a \leq 150 \AA$; Draine 1985), it is essential for further growth through coagulation.

Finally, note that at low densities $\left(n_{0}<10^{3} \mathrm{~cm}^{-3}\right)$ the relative grain velocities will be larger than $10^{5} \mathrm{~cm} \mathrm{~s}^{-1}$. In that regime shattering rather than coagulation will dominate. Since (again) the grain-grain collision rate is much faster than the dynamical evolution timescale of a molecular cloud, the grain size distribution can be substantially altered, leading to an increased population of small grains.

\section{STAR FORMATION AND INTERSTELLAR DUST}

Many protostars have associated strong winds. In principle, dust condensation in this wind may be of some importance for interstellar dust. For a typical mass loss rate of $10^{-6} \mathrm{M}_{\odot} \mathrm{yr}^{-1}$, a dynamical lifetime of $10^{5} \mathrm{yr}$, a star formation efficiency of $2 \%$ and a typical stellar mass of $1 \mathrm{M}_{\odot}$ (Edwards and Snell, 1984; Margulis and Lada, 1985; Shu et al., 1987), at most $2 \times 10^{-3}$ of all the available heavy elements may have been converted into grains in a molecular cloud. Although over the cycling timescale between stars and the ISM, about $20 \%$ of all gas phase species may have been converted into solid grains this way - comparable to late type giants (cf., Tielens, 1988; Gehrz, 1989) - such a high efficiency is quite unlikely. However, for 
a molecular cloud such as $\rho$ Oph, which seems to be forming a bound star cluster (Wilking and Lada, 1983), the star formation efficiency is much higher and thus locally and recently produced dust may be more important.

Although locally produced star formation dust is probably not a dominant interstellar dust component, it can be a particularly important source of minor interstellar dust components, in particular, if the mass loss originates in, or entraines, a considerable amount of material from a protoplanetary disk surrounding the protostar. In this respect, it should be kept in mind that the inner solar system seems to have lost its complement of $H$ and $H e$, presumably through a strong stellar wind during an active $T$ Tauri phase. It is quite likely that some protosolar dust has been ejected as well at that stage. Since dust particles in these disks do not settle to the midplane until they have grown to sizes in the range of $10 \mathrm{~cm}$, this process may have pronounced influence on the large grain component in the ISM. Likewise, iron is expected to be an important grain condensate at the inner boundary of the protoplanetary disk. Iron grains as well as large grains may have a pronounced influence on the far-IR and submillimeter dust opacity. This should be kept in mind when converting observed dust column densities into gas masses, in particular around protostars.

Coagulation processes have played an important role in the formation of planetary and cometary bodies in the protoplanetary disk around the early sun. This accretionary record has been preserved to some extent in Interplanetary Dust Particles (IDPs) and comets. The IDPs are of particular interest since the scale-size of their building blocks is quite similar to that of interstellar grains and thus their study might reveal insight in the physics of interstellar grain collisions. Moreover, the presence of a deuterium enriched phase, associated with a carbonaceous carrier, is generally taken to imply the presence of a largely, chemically unmodified interstellar component (cf., Kerridge, 1989). IDPs can have distinctly different structures (Bradley and Brownlee, 1986; see Sandford, 1989). Collisional coagulation leads to a very open and fluffy structure (cf., Fig. 10). A typical example of such a fluffy IDP, which presumably reflect best the coagulation processes taking place in the planetary disk, is shown in Fig. 11. This IDP is an agglomerate of (sub) $\mu$ m-sized mineral grains enveloped or embedded in a seemingly continuous carbonaceous mantle. This structure is quite unlike the structure of grains coagulated in the laboratory (cf., Fig. 10).

One possible model that might perhaps explain such a structure is that of coagulation of mineral grains coated with thick, irradiated ice mantles (Johnson and Lanzerotti 1986). The diffusion of the trapped radicals, resulting from the collisional heating, may have lead to an organic refractory grain mantle which "glues" the individual mineral grains together (cf., §4). Simultaneously, the released reaction heat may have caused the evaporation of the icy molecules. Further processing through warm-up, and UV, solar wind, or cosmic ray irradiation may have transformed this organic mantle into amorphous carbon. It should be emphasized that, even if this model is correct, there is presently no evidence linking these processes to the interstellar medium rather than the protoplanetary disk. 


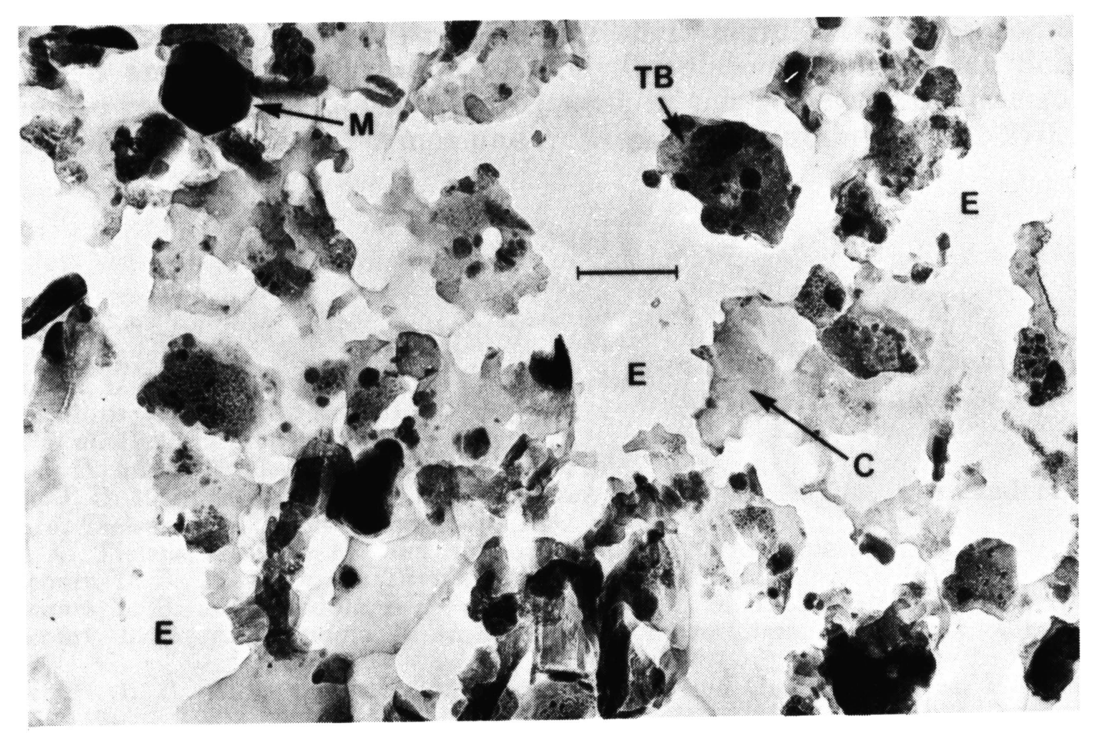

Fig. 11. Electron micrograph of a thin sectioned, very fluffy, interplanetary dust particle (Bradley and Brownlee, 1986). Before sectioning the pores of this IDP have been filled with epoxy (labeled E). Note the individual mineral grains (labeled $M$ ), which are "glued" into a much larger agglomerate by a seemingly continuous carbonaceous matrix (labeled C). Tar bals (TB) consist of a compact clump of small mineral grains embedded in a carbon matrix.

\section{SUMMARY}

We have discussed the properties of dust in dense clouds with an emphasis on grain growth. Observations clearly show that grain growth is an important process in such regions. Although there is direct observational evidence for the formation of icy grain mantles, they cannot account for the observed large increases in the ratio of selective to total extinction and in the wavelength of maximum polarization. In particular, accretion leads to a mantle thickness which is independent of grain size. Since small grains dominate the total grain surface area, the average increase in grain size of the large grains is relatively small ( $\lesssim 150 \AA$; Draine, 1985$)$ and visual and NIR extinction and polarization should not be affected that much by grain mantle accretion. There is no such constraint on the increase in grain size due to coagulation. However, coagulation by itself occurs only at extremely high densities and is therefore generally unimportant. Synergisme of these two processes, icy grain mantle formation and coagulation, may actually hold the key to the observed increases in grain size inside dense clouds. Ice mantle growth will lead to better sticking of two colliding grains. Coagulation will lead to a decrease of the small grain population and, thus, to thicker ice mantles. Moreover, the same collision that leads to coagulation may also initiate radical diffusion in an irradiated icy grain mantle and thereby add to the formation of a refractory grain mantle, enveloping and binding both grains. 


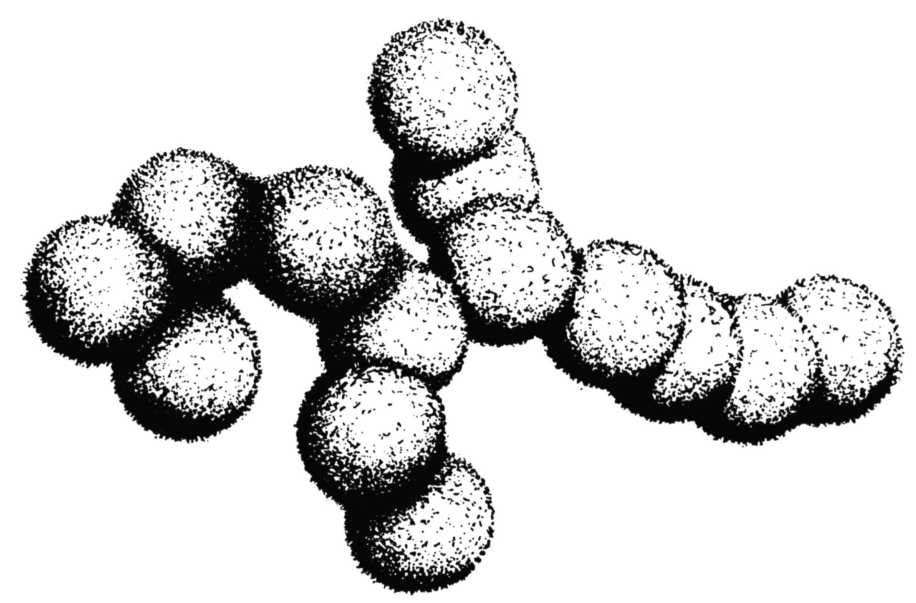

Fig. 12. Interstellar dust is proposed to consist of small ( $\lesssim 500 \AA)$ grains "glued" together in large, open conglomerates $(\approx 3000 \AA)$ by an all enveloping carbonaceous mantle. The mantle may be quite thin as sketched here or might dominate the volume as for the IDP shown in Fig. 11, depending on the relative importance of accretion and coagulation.

Thus a model for interstellar dust is proposed in which small grains are "glued" together in large, open conglomerates by an all enveloping carbonaceous grain mantle (cf., Fig. 12). In this model a continuous cycling of matter is envisioned between small and large grains through coagulation in clouds and shattering in shocks and between gas and solid phase through accretion in clouds and grain destruction in shocks. It should be emphasized that presently there is little direct observational support for the open interstellar grain structures sketched in Fig. 12. As for most interstellar dust models, the particular properties adopted here merely reflect the author's preoccupation with certain theoretical arguments. The morphological structure of interstellar grains in this model resemble the open IDP structure shown in Fig. 11. Such a conglomerate will be quite strongly bonded. In this respect, it is important to realize that grain agglomerates bonded only by volatile ices or by weak van der Waals forces will not survive long in the harsh environment of the diffuse interstellar medium. Thus, if grain growth through coagulation or ice mantle accretion in dense clouds is to have a lasting effect on the dust in the diffuse interstellar medium, photoprocessing has to convert the ice into more refractory "glue".

ACKNOWLEDGEMENTS. The 5-8 $\mu \mathrm{m}$ observations shown in Fig. 8 have been made possible by the dedication and hard work of the KAO crew and support staff. The observations reported in Fig. 6 have been obtained with the CGAS spectrometer developed by Alan Tokunaga at the Infrared Telescope Facility under 
contract from the National Aeronautics and Space Administration. I thank Dr. Iijima and Drs. Bradley and Brownlee for their permission to reproduce Figs. 10 and 11. I am also very grateful to Drs. Pendleton, Chokshi and Schutte for permission to report some of their (hard) work in this review. This was supported with funds allotted by NASA-Ames under interchange number NCA2-270.

\section{REFERENCES}

Allamandola, L. J., 1984 in Galactic and Extragalactic IR Spectroscopy, eds. M. F. Kessler and J. P. Phillips, (Dordrecht: Reidel), p. 5.

Baas, F. et al. 1989, in preparation.

Bradley, J. P., and Brownlee, D. E. 1986, Science, 281, 1542.

Bregman, J. D. 1989, in IAU Symposium 135, Interstellar Dust, eds. L. J. Allamandola and A. G. G. M. Tielens, (Dordrecht: Kluwer), p. 109.

Chokshi, A., Tielens, A. G. G. M., and Hollenbach, D., 1989, in preparation.

d'Hendecourt, L. B., 1984, Ph. D. Thesis, RijksUniversiteit Leiden.

d'Hendecourt, L. B., Allamandola, L. J., and Greenberg, J. M. 1985, Astr. Ap., $152,130$.

d'Hendecourt, L. B., Allamandola, L. J., Baas, F., and Greenberg, J. M. 1982, Astr. Ap., 109, L12.

d'Hendecourt, L. B., Allamandola, L. J., Grim, R. J. A., and Greenberg, J. M. 1986, Astr. Ap., $158,119$.

Dalgarno, A., and Lepp, S. 1985, Ap. J. (Letters), 287, L47.

de Boer, K.S., Lenhart, H., van der Hucht, K.A., Kamperman, T.M., Kondo, Y., and Bruhweiler, F. C., 1986,Astr. Ap.157, 119.

Draine, B. T. 1985, in Protostars and Planets II, eds. D. Black and M. Mathews, (Tucson: Univ. Arizona Press).

Draine, B. T., and Lee, H. M. 1984, Ap. J., 285, 89.

Edwards, S., and Snell, R. L. 1984, Ap. J., 281, 237.

Jura, M., 1980, Ap. J., 285, 63.

Geballe, T. R. 1986, Astr. Ap., $162,248$.

Gehrz, R. D. 1989, in IAU Symposium 195, Interstellar Dust, eds. L. J. Allamandola and A. G. G. M. Tielens, (Dordrecht: Kluwer), p. 445.

Greenberg, J. M. 1979, in Stars and Stellar Systems, ed. B. Westerlund, (Dordrecht: Reidel), p. 173.

Grim, R. J. A. 1988, Ph. D. Thesis, RijksUniversiteit Leiden.

Grim, R. J. A., and d'Hendecourt, L. B. 1986, Astr. Ap., 167, 161.

Grim, R. J. A., and Greenberg, J. M. 1987, Ap. J. (Letters), \$21, L91.

Hagen W., and Tielens, A. G. G. M. 1981, J. Chem. Phys., 75, 4198.

Hagen, W., Tielens, A. G. G. M., and Greenberg, J. M. 1983, Astr. Ap., 117, 132.

Hollim, P., and Pritchard, J. 1980, in Vibrational Spectroscopy for Adsorbed Species, eds. A. T. Bell and M. L. Hair, (New York: Acad. Press), p. 51.

lijima, S. 1987, Japan J. Appl. Phys., 26, 365.

Jenkins, E. 1989, in IAU Symposium 195, Interstellar Dust, eds. L. J. Allamandola and A. G. G. M. Tielens, (Dordrecht: Kluwer), p. 23.

Johnson, R. E., and Lanzerotti, L. J., 1986, Icarus, 66, 619.

Kendall, K. 1980, Contemp. Phys., 21, 277.

Kerridge, J. F. 1989, in IAU Symposium 195, Interstellar Dust, eds. L. J. Allamandola and A. G. G. M. Tielens, (Dordrecht: Kluwer), p. 383.

Knacke, R. F., and McCorkle, S. M. 1987, A. J., $94,972$.

Knacke, R. F., McCorkle, S. M., Puetter, R. C., Erickson, E. F., and Kratschmer, W. 1982, Ap. J., $260,141$.

Lacy, J. H. et al., 1984, Ap. J., 276, 533.

Larson, H. P., Davis, D. S., Black, J. H., and Fink, U. 1985, Ap. J., 299, 873.

Léger, A., Jura, M., and Omont, A. 1985, Astr. Ap., 144, 147.

Margulis, M., and Lada, C. J. 1985, Ap. J., 299, 925.

Mathis, J. S., Rumpl, W., and Nordsieck, K. H. 1977, Ap. J., $217,425$.

McKee, C. F. 1989, in IAU Symposium 195, Interstellar Dust, eds. L. J. Allamandola and A. G. G. M. Tielens, (Dordrecht: Kluwer), p. 431. 
Merrill, K. M., Russell, R. W., and Soifer, B. T. 1976, Ap. J., $207,763$.

Myers, P. C. 1987 in Interstellar Processes, eds. D. Hollenbach and H. A. Thronson, Jr., (Dordrecht: Reidel), p. 71.

Newton, I. 1686, Principia, (London: Dover).

Nuth, J. A., and Moore, M. H. 1988, Ap. J. (Letters), 829, L113.

Pendleton, Y. 1987, Ph. D. Thesis, UC Santa Cruz.

Pendleton, Y., Tielens, A. G. G. M., and Werner, M. W. 1989, in preparation.

Prasad, S. S., and Tarafdar, S. P. 1983, Ap. J., 267603.

Rossler, K., 1986, Rad. Eff., 99, 21.

Rouan, D., and Léger, A. 1984, Astr. Ap., 182, L1.

Sandford, S. A. 1989, in IAU Symposium 195, Interstellar Dust, eds. L. J. Allamandola and A. G. G. M. Tielens, (Dordrecht: Kluwer), p. 403.

Sandford, S. A., Allamandola, L. J., Tielens, A. G. G. M., and Valero, G. J. 1988, Ap. J., s29, 498.

Scalo, J. M. 1977, Astr. Ap., 55, 253.

Schutte, W. 1988, Ph. D. Thesis, RijksUniversiteit Leiden.

Serkowski, K., Mathewson, D. S., and Ford, V. L. 1975, Ap. J., 196, 261.

Shu, F., Lizano, S., and Adams, F. 1987, Ann. Rev. Astr. Ap..

Shull, J. M., and van Steenbergen, M. E. 1985, Ap. J., 294, 599.

Sternberg, A., Dalgarno, A., and Lepp, S. Ap. J., \$20, 676.

Tielens, A. G. G. M. 1983, Astr. Ap., 119, 177.

1988, in Carbon in the Galaxy: Studies from Earth and Space, ed. J. Tarter, NASA CP-, in press.

Tielens, A. G. G. M., and Allamandola, L. J. 1987a, in Physical Processes in Dense Clouds, eds. G. E. Morfill and M. Scholer, (Dordrecht: Reidel), p. 333.

- $1987 \mathrm{~b}$, in Interstellar Processes, eds. D. Hollenbach and H. Thronson, (Dordrecht: Reidel), p. 397 .

Tielens, A. G. G. M., Allamandola, L. J., Bregman, J., Goebel, J., d'Hendecourt, L. B., and Witteborn, F. C'1984, Ap. J., $287,697$.

Tielens, A. G. G. M., and Hagen, W. 1982, Astr. Ap., 114, 245.

Tielens, A. G. G. M., Tokunaga, A., Geballe, T. R., and Baas, F. 1989, in preparation.

van de Bult, C. E. P. M. 1982, unpublished.

Volk, H. J., Jones, F. C., Morfill, G. E., and Roser, S. 1980, Astr. Ap., 85, 316.

Werner, M. W., Dinerstein, H. L., and Capps, R. W. Ap. J. (Letters), 265, L13.

Whittet, D. C. B. and van Breda, I. G, 1978, Astr. Ap., 66, 57.

Wilking, B. A., and Lada, C. J. 1983, Ap. J., 274, 698. 